\title{
Taxol Interferes with the Interaction of Microtubule-Associated Proteins with Microtubules in Cultured Neurons
}

\author{
Mark M. Black \\ Department of Anatomy, Temple University School of Medicine, Philadelphia, Pennsylvania 19140
}

\begin{abstract}
Treatment of neurons with taxol leads to the formation of microtubule bundles in which individual microtubules are much more closely spaced than in untreated neurons (Letourneau and Ressler, 1984). This suggests that taxol interferes with the mechanisms that regulate microtubule spacing in situ. I have determined whether treatment of neurons with taxol alters the composition of their microtubules, reasoning that such alterations may be related to the taxolinduced alterations in microtubule spacing. Cultures of sympathetic neurons were incubated with ${ }^{35}$ S-methionine and either taxol, podophyllotoxin, a potent microtubule-depolymerizing agent, or dimethyl sulfoxide (DMSO), the solvent for the drugs. The levels of labeled microtubule-associated proteins (MAPs) assembled into microtubules in the various cultures were then assayed biochemically. I focused on 4 MAPs: tau, chartins, MAP-2, and the MAP with a molecular mass of $210,000 \mathrm{Da}(210 \mathrm{kDa})$. In untreated cultures, these MAPs are prominent components of microtubules. The levels of all MAPs, as well as tubulin, in microtubules were greatly reduced in cultures treated with podophyllotoxin, compared to controls. Taxol had varied effects on the interaction of MAPs with microtubules in situ. Microtubules formed in the presence of taxol contained normal or slightly elevated levels of tau and the $210 \mathrm{kDa}$ MAP compared to microtubules in control cultures. In contrast, microtubules formed in the presence of taxol were almost completely devoid of chartin MAPs and MAP-2 compared to controls. These results show that taxol interferes with the interaction of some, but not all, MAPs with microtubules in situ. The altered MAP composition of microtubules in taxol-treated neurons may contribute to the abnormal spacing of microtubules seen in such neurons.
\end{abstract}

The spacing between neighboring microtubules is tightly controlled in neurons. This is particularly well illustrated by the fact that, within individual neurons, the distance between neighboring microtubules in axons is, on average, much less than that in dendrites (Wuerker and Kirkpatrick, 1972; Bartlett and Banker, 1984). This regional variation in microtubule spacing may involve the class of microtubule proteins known as micro-

Received Feb. 24, 1987; revised Apr. 27, 1987; accepted May 20, 1987.

I would like to thank Dr. Lester Binder for providing antibodies to tau and MAP-2, Drs. John Aletta and Lloyd Greene for sharing their unpublished data, and the National Products Branch of NCI for providing taxol. The work reported here was supported by NIH Grant NS17681. Dr. Black is also the recipient of a Research Career Development Award from NIH.

Correspondence should be addressed to Dr. Mark M. Black, Department of Anatomy, Temple University School of Medicine, 3420 Broad Street, Philadelphia, PA 19140

Copyright (C) 1987 Society for Neuroscience $0270-6474 / 87 / 113695-08 \$ 02.00 / 0$ tubule-associated proteins (MAPs). First, under in vitro conditions, MAPs influence the spacing between microtubules (Herzog and Weber, 1978; Kim et al., 1979; Vallee and Davis, 1983; Brown and Berlin, 1985), presumably by virtue of projection domains that extend away from the surface of the tubulin lattice (Zingsheim et al., 1979; Langford, 1983). Moreover, MAPs appear to differ quantitatively with regard to their effect on the spacing between microtubules. For example, MAP-2-containing microtubules are more widely separated than tau-containing microtubules (Herzog and Weber, 1978; Black, 1987). Second, axonal microtubules contain different MAPs than do dendritic microtubules (Caceres et al., 1984; DeCamilli et al., 1984; Huber and Matus, 1984; Binder et al., 1985; Peng et al., 1986). Specifically, tau and the $M_{\mathrm{r}} 210,000$ Da MAP are major components of axonal microtubules, but not of dendritic microtubules, while MAP-2 is a major associated protein of dendritic, but not axonal, microtubules.

Several recent observations suggest that the drug taxol, a potent microtubule-stabilizing agent (Schiff and Horwitz, 1980), may be useful as a probe of MAP contribution to microtubule spacing in situ. In taxol-treated neurons, microtubules are organized into compact bundles in which the individual microtubules are much more closely packed than thcy arc in untreated neurons (Masurovsky et al., 1983; Letourneau and Ressler, 1984). Thus, one apparent effect of taxol is to override the normal mechanisms that regulate microtubule spacing in situ. I have taken advantage of this property of taxol to determine whether the composition of microtubules in taxol-treated neurons differs from that in untreated neurons, reasoning that such differences, if they exist, may be related to the abnormal spacing between microtubules observed in taxol-treated neurons. The results show that taxol interferes with the interaction of some, but not all, MAPs with microtubules. Portions of these results have been published previously in preliminary form (Black and Peng, 1985).

\section{Materials and Methods}

Cell culture and metabolic labeling. Dissociated cultures of rat sympathetic neurons were prepared as described previously (Peng et al., 1985) and used after 7-14 $\mathrm{d}$ in culture; mitotic poisons were used to eliminate non-neuronal cells. Cultures were labeled with ${ }^{35} S$-methionine for 18$24 \mathrm{hr}$ in medium containing $10 \%$ of the normal amount of methionine. For some experiments, cultures were labeled overnight, fed with fresh complete medium, and then incubated an additional $18-48 \mathrm{hr}$.

Drug treatments. Cultures were treated for $18-24 \mathrm{hr}$ with $10 \mu \mathrm{M}$ taxol, $4.4 \mu \mathrm{M}$ podophyllotoxin, or just with dimethyl sulfoxide (DMSO), the solvent for these drugs, at $0.1 \%$ (vol/vol) final concentration. Podophyllotoxin was obtained from Sigma Chemical Co., and taxol was a gift from the National Products Branch, National Cancer Institute. Appropriate volumes of 1000 times concentrated stock solutions were added to the cultures to achieve the desired concentration. In most experiments, drug treatments were coincident with metabolic labeling. 
However, for some experiments, cultures were labeled first and then treated with the drugs.

Cell extractions. A sequential extraction procedure was used to prepare fractions enriched in assembled microtubule proteins. The procedure is described in detail in Black et al. (1984) and is modified from Solomon et al. (1979). In brief, control and drug-treated cultures are extracted with a microtubule-stabilizing buffer $\left[0.1 \mathrm{M}\right.$ piperazine $\cdot N-N^{\prime}$ bis(2-ethanesulfonic acid) (PIPES), pH 6.9, 2 M glycerol, $1 \mathrm{~mm} \mathrm{MgSO}_{4}$, $2 \mathrm{mM}$ EGTA, protease inhibitors] containing $0.2 \%$ (wt/vol) Triton X-100; the drugs were not included in the extraction buffer. This extraction procedure solubilized unassembled microtubule proteins, while assembled microtubule proteins remained with the Triton X-100-insoluble cytoskeleton. These cytoskeletons were then suspended in a buffer containing $5 \mathrm{mM} \mathrm{Ca}^{2+}$, incubated on ice for $10-20 \mathrm{~min}$, and then centrifuged at $12,000 \times g$ for $10 \mathrm{~min}$ at $4^{\circ} \mathrm{C}$, to yield a cold $/ \mathrm{Ca}^{2+}$-soluble fraction. This treatment depolymerized most of the microtubules in the cytoskeletons and thereby solubilized their proteins. The cold $/ \mathrm{Ca}^{2+}$-insoluble fraction also contains tubulin, but this represents a comparatively small portion of the total cytoskclctal tubulin in the neuron (Black et al., 1984). Thus, the cold $/ \mathrm{Ca}^{2+}$-soluble fractions contain most of the proteins assembled into microtubules at the time of cell lysis, and will be designated microtubule fractions. The microtubule fractions and, when appropriate, the cold $/ \mathrm{Ca}^{2+}$-insoluble fractions from the various cultures were assayed by gel electrophoresis and fluorography.

Other procedures. Immunoprecipitation was performed as described in Peng et al. (1985). The antibodies used were mouse monoclonals against MAP-2 or tau. These antibodies were generously provided by Dr. Lester Binder, Department of Cell Biology and Anatomy, University of Alabama, Birmingham. The properties of these antibodies are described in Caceres et al. (1984) and Binder et al. (1985).

One-dimensional SDS (1D) and 2-dimensional isoelectric focusing $\times$ SDS (2D)-PAGE were performed as described in Black et al. (1984) and Peng ct al. (1985). Labclcd protcins in gels werc visualized by fluorography (Bonner and Laskey, 1974). Quantitation of labeled proteins solubility from 2D gels was as described in Black et al. (1986b).

\section{Results}

The present studies determined whether treatment of intact neurons with taxol altered the composition of MAPs assembled onto microtubules. Fractions enriched in assembled microtubule proteins were prepared from untreated cultures, from cultures depleted of microtubules by treatment with $4.4 \mu \mathrm{M}$ podophyllotoxin, from cultures treated with $10 \mu \mathrm{M}$ taxol, or with $0.1 \%$ DMSO (the solvent for the drugs). The MAPs in microtubule fractions from taxol-treated cultures were compared to those in the corresponding fractions from untreated, podophyllotoxin- and DMSO-treated cultures. If taxol interferes with the incorporation of a given MAP into microtubules, then that MAP will be diminished or absent from microtubule fractions prepared from taxol- as well as podophyllotoxin-treated cultures, but will be present in microtubule fractions from the various control (i.e., untreated and DMSO-treated) cultures. On the other hand, if taxol enhances the incorporation of a MAP into microtubules, then that MAP will be more abundant in microtubule fractions prepared from taxol-treated cultures than in control cultures.

I have examined the following neuronal MAPs: tau, MAP-2, a MAP with an apparent molecular mass of $210,000 \mathrm{Da}$, and a family of proteins ranging in apparent molecular mass from 64,000 to $80,000 \mathrm{Da}$. These proteins are biochemically and immunologically distinct from tau (Magendantz and Solomon, 1985; Peng et al., 1985). They resemble a similar family of MAPs, designated chartins (Magendantz and Solomon, 1985), in neuroblastoma and PC12 pheochromocytoma cells in mobility in $2 \mathrm{D}$ gels and $1 \mathrm{D}$ fragment patterns generated by partial proteolysis with $S$. aureus protease (see Pallas and Solomon, 1982; Black and Kurdyla, 1983; Black et al., 1986a; and data not shown). I will therefore refer to these neuronal MAPs as chartins.

\section{Taxol interferes with the incorporation of chartins into microtubules}

Figure 1 shows representative fluorographs depicting the labeled peptides present in microtubule fractions prepared from the various cultures. In this analysis, taxol, podophyllotoxin, and DMSO treatments were coincident with the labeling period. As expected, $\alpha$ - and $\beta$-tubulin (Fig. 1a, arrows) are major components of microtubule fractions from untreated, taxol-, and DMSO-treated cultures, but are greatly diminished in the microtubule fractions prepared from cultures treated with podophyllotoxin. The tubulin of podophyllotoxin-treated cultures partitions mostly with the Triton X-100-soluble fraction (Black et al., 1986b). Note also that, as expected, the chartins (Fig. la, arrowheads) are absent from microtubule fractions from podophyllotoxin-treated cultures, compared to control cultures. These MAPs are also greatly decreased in microtubule fractions prepared from taxol-treated cultures, but are present at control levels in microtubule fractions prepared from DMSO-treated cultures. These latter findings are particularly apparent in the 2D gel analyses shown in Figure $1, b, c$. We did not detect any labeled bands in microtubule fractions from taxol-treated cultures that were not also present in the corresponding fractions from DMSO-treated or untreated cultures (see Figs. 1, 5).

It is important to note that while the microtubule fractions are enriched in proteins assembled onto microtubules, they contain many non-microtubule, or background, proteins (Solomon et al., 1979; Black and Kurdyla, 1983). These background proteins serve as an important reference for evaluating the similarity in fluorographic exposures of control and drug-treated samples. If the exposures are comparable, then the background bands or spots in the various samples should be of equal intensity. As can be seen in Figure 1, and also in Figures 3-5, such equality has been obtained. Thus, visual comparison provides an accurate means of assessing the relative levels of chartins in microtubule fractions from the various cultures.

Among the possible explanations for the absence of chartins from microtubule fractions from taxol-treated cultures are that (1) taxol inhibits the synthesis and/or enhances the degradation of the chartins, thereby reducing the levels of labeled chartins available for incorporation into microtubules; (2) taxol treatment renders microtubules that contain the chartins stable during treatment with $\mathrm{Ca}^{2+}$ at $0^{\circ} \mathrm{C}$; and (3) taxol interferes with the incorporation of chartins into microtubules. Several experiments have been performed to evaluate these possibilities.

Figure 2 shows representative fluorographs of whole cell SDS extracts of cultures treated with taxol or DMSO for $24 \mathrm{hr}$. The overall pattern of labeled proteins observed in the taxol-treated material is very similar to that in the DMSO-treated material. In particular, the levels of labeled tubulin $(\mathrm{T})$ and the chartins (arrows and arrowheads) closely resemble each other, indicating that taxol treatment has minimal effects on the accumulation of labeled chartins in cultured neurons.

To evaluate whether taxol treatment renders microtubules containing chartins stable during combined exposure to cold and millimolar $\mathrm{Ca}^{2+}$, microtubule-containing cytoskeletons from DMSO- and taxol-treated cultures were fractionated into cold/ $\mathrm{Ca}^{2+}$-soluble and -insoluble material. In DMSO-treated cultures, $\sim 75 \%$ of the tubulin is cytoskeletal-associated (Black et al., 


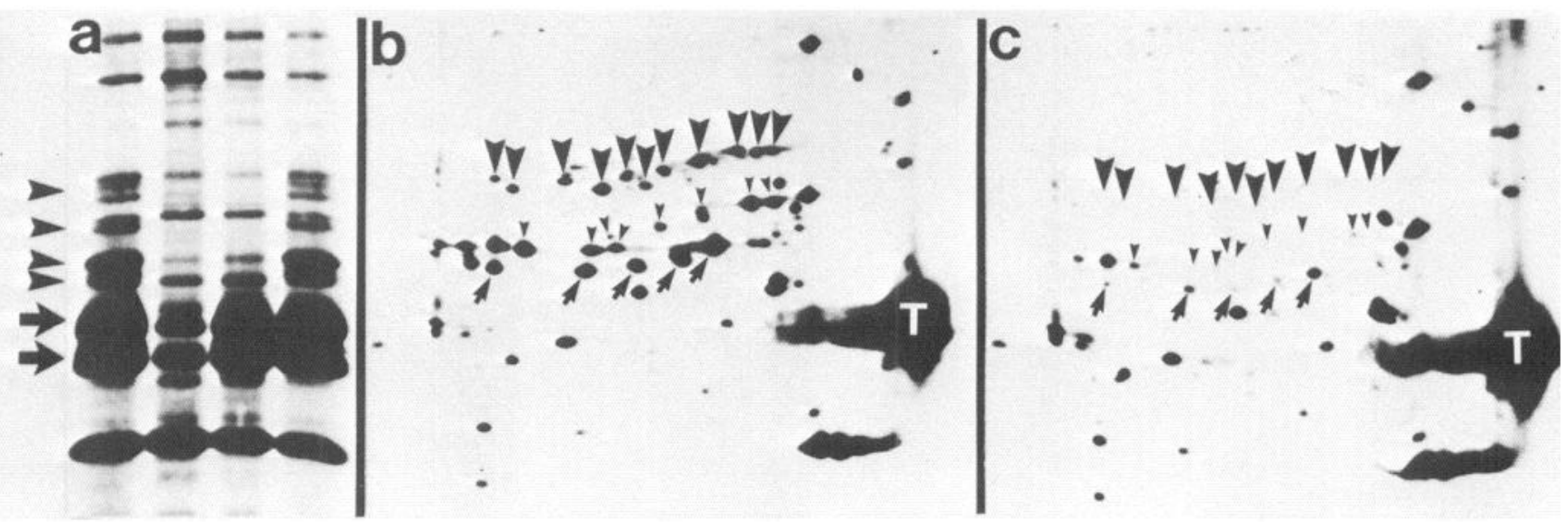

Figure 1. Comparison of the effects of taxol and podophyllotoxin treatment on the presence of chartins in microtubule fractions. Cultures were labeled with ${ }^{35} \mathrm{~S}$-methionine for $18 \mathrm{hr}$, and coincident with the labeling period they were also treated with $4.4 \mu \mathrm{M}$ podophyllotoxin, $10 \mu \mathrm{M}$ taxol, $0.1 \%$ DMSO, or nothing. Microtubule fractions were prepared from the cultures as described in Materials and Methods, and analyzed by gel electrophoresis and fluorography. The data shown are representative of 6 separate experiments. $a$, One-dimensional gel analysis showing, from left to right, the labeled material from untreated, podophyllotoxin-treated, taxol-treated, and DMSO-treated cultures. Tubulin (arrows) is very prominent in microtubule fractions from all but the podophyllotoxin-treated culture. The chartins (arrowheads) are prominent in the material prepared from control and DMSO-treated cultures, but are absent or greatly diminished in the corresponding material prepared from podophyllotoxin- and taxoltreated cultures. $b, c$, Two-dimensional gel analyses of microtubule fractions prepared from DMSO-treated $(b)$ and taxol-treated $(c)$ cultures (the fluorographs were exposed for $14 \mathrm{~d}$ ). In $2 \mathrm{D}$ gels, each chartin resolves into a set of peptides that range considerably in pI and also in apparent molecular mass (Black and Kurdyla, 1983). Within each molecular-weight class, all of the variants are indistinguishable by peptide mapping. The chartins are indicated by various symbols in $b$, and the expected positions of the chartins are indicated in $c$. The arrows depict the $64 \mathrm{kDa}$ chartins (apparent $M_{\mathrm{r}} \mathrm{s}, 60-64 \mathrm{kDa}$ ), the small arrowheads identify the $68 \mathrm{kDa}$ chartins (apparent $M_{\mathrm{r}} \mathrm{s}, 64-68 \mathrm{kDa}$ ), and the large arrowheads identify the $80 \mathrm{kDa}$ chartins (apparent $M_{\mathrm{r}} \mathrm{s}, 76-80 \mathrm{kDa}$ ). The chartins are quite prominent in the material from DMSO-treated cultures, but are greatly diminished in the material from taxol-treated cultures.

1986b), the majority of which is cold/ $\mathrm{Ca}^{2+}$-soluble (Fig. 3, $a, b$ ). The distribution of chartins between soluble and cytoskeletal fractions is indistinguishable from that in untreated cultures (see Black and Kurdyla, 1983, for quantitative data; and data not shown), and the cytoskeletal-associated chartins partition almost quantitatively with the cold/ $\mathrm{Ca}^{2+}$-soluble fraction (Fig. 3, $a, b)$. In taxol-treated cultures, $95-98 \%(n=3)$ of the tubulin partitions with the cytoskeleton. Thus, taxol treatment enhances
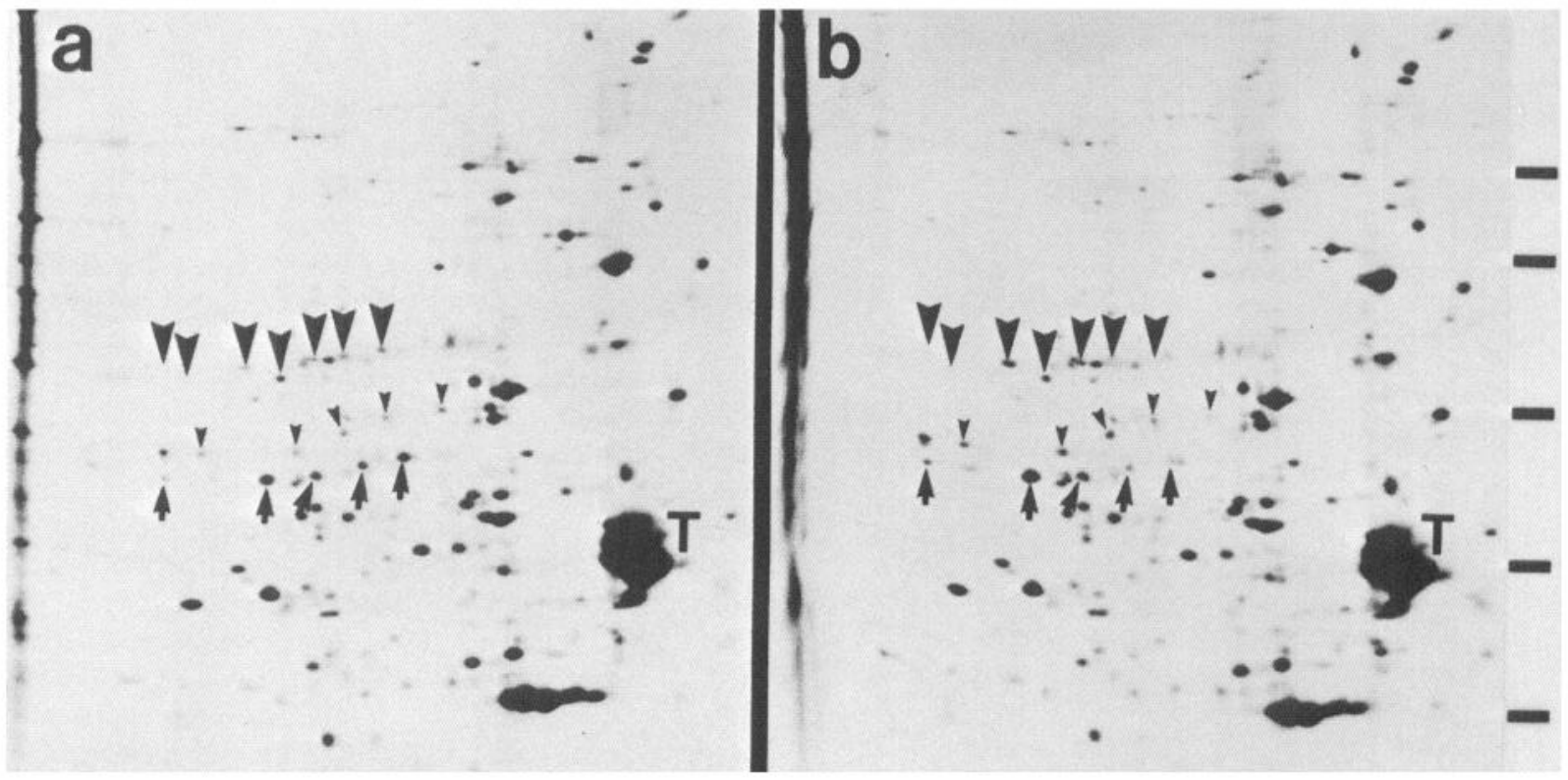

Figure 2. Analysis of labeled chartins in whole cell SDS extracts of sister cultures treated with either DMSO $(a)$ or taxol $(b)$. Cultures were labeled for $20 \mathrm{hr}$ and drug treatment was coincident with the labeling period. Cultured neurons were dissolved directly in SDS-containing sample buffer and analyzed by $2 \mathrm{D}$ gel electrophoresis and fluorography ( $2 \mathrm{~d}$ exposure). T, Tubulin. The chartins are indicated as in Figure 1 . The 64 and $68 \mathrm{kDa}$ chartin species are well visualized; only some of the $80 \mathrm{kDa}$ chartins could be identified. Note that while the total levels of labeled tubulin and chartins are comparable in DMSO- and taxol-treated cultures, the pattern of chartin variants differs between the cultures. This is particularly clear for the $64 \mathrm{kDa}$ chartins (see text for additional discussion). 


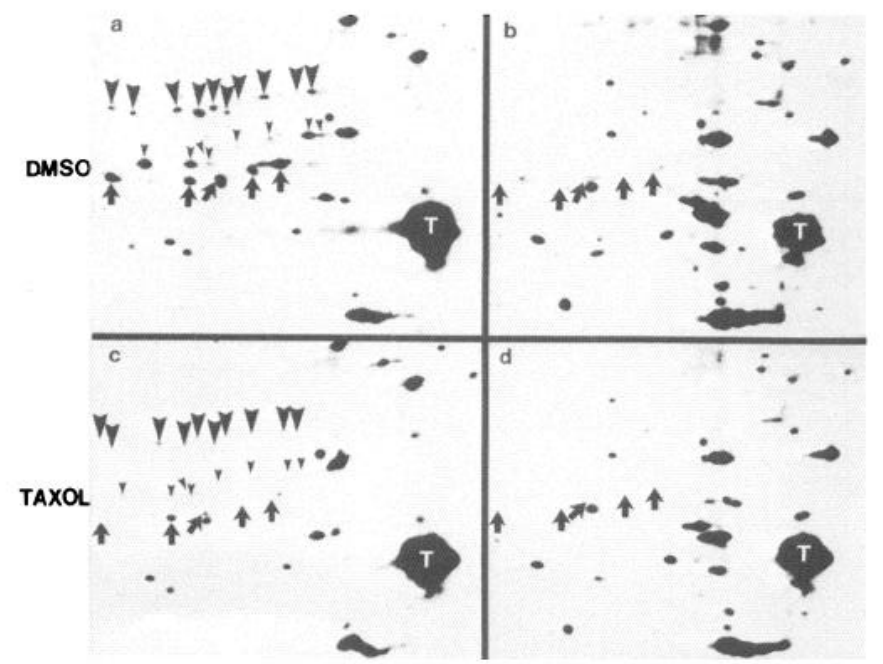

Figure 3. Effects of taxol treatment on the $\mathrm{Ca}^{2+}$ solubility of chartins. Sister cultures were labeled for $24 \mathrm{hr}$ and also treated with either taxol or DMSO during the labeling period. Triton X-100-insoluble cytoskeletons were prepared from the cultures and extracted with $5 \mathrm{mM} \mathrm{Ca}^{2+}$ at $0^{\circ} \mathrm{C}$, as described in Materials and Methods. Cold $/ \mathrm{Ca}^{2+}$-soluble and -insoluble fractions were prepared and analyzed by 2D gel electrophoresis and fluorography ( $7 \mathrm{~d}$ exposures were used for all fluorographs). $a, b$, Labeled material in the cold/ $\mathrm{Ca}^{2+}$-soluble and -insoluble material, respectively, from the DMSO-treated culture. $c, d, \mathrm{Cold} / \mathrm{Ca}^{2+}$-soluble and -insoluble material, respectively, from the taxol-treated culture. In $a$ and $c$, the chartins or their expected positions are indicated as described in the legend to Figure 1 . In $b$ and $d$, the expected positions of the $64 \mathrm{kDa}$ chartins are indicated. Note that the chartins are prominent in the $\mathrm{Ca}^{2+}$-soluble material from DMSO-treated, but not taxol-treated cultures. These MAPs are only trace components in the insoluble fraction from these cultures. The data shown are representative of $4 \mathrm{ex}$ periments.

the levels of cytoskeletal-associated tubulin in intact neurons. Most of this tubulin is also cold/ $\mathrm{Ca}^{2+}$-soluble (Fig. 3, $c, d$ ). Note that taxol treatment resulted in only a modest increase in cold/ $\mathrm{Ca}^{2+}$-insoluble tubulin. Taxol microtubules assembled in vitro show a similar lability to combined exposure to cold and $\mathrm{Ca}^{2+}$ (Collins and Vallee, 1986). The trace levels of labeled chartins in cytoskeletons from taxol-treated cultures partitioned entirely with the cold/ $\mathrm{Ca}^{2+}$-soluble fraction (Fig. 3, c, d). The remainder of the chartins of taxol-treated cultures partitioned with the Triton X-100-soluble fraction (data not shown).

The most straightforward interpretation of the above-de-

Table 1. Relative abundances of the $64 \mathrm{kDa}$ chartin species in DMSO- and taxol-treated neurons

Relative abundance of $64 \mathrm{kDa}$ chartin variants

\begin{tabular}{llllll}
\cline { 2 - 6 } Treatment & $\mathrm{a}$ & $\mathrm{b}$ & $\mathrm{c}$ & $\mathrm{d}$ & $\mathrm{e}$ \\
\hline DMSO & 0.09 & 0.42 & 0.22 & 0.14 & 0.12 \\
DMSO & 0.06 & 0.45 & 0.20 & 0.14 & 0.14 \\
Taxol & 0.07 & 0.70 & 0.15 & 0.06 & 0.02 \\
Taxol & 0.07 & 0.71 & 0.14 & 0.05 & 0.03
\end{tabular}

The relative abundances of the 5 variants that comprise the $64 \mathrm{kDa}$ chartin family were quantified from $2 \mathrm{D}$ gels like those of Figure 2 . The 5 variants were designated a-e, proceeding from the most basic (a) to the most acidic (e) (see Fig. 2). The spots corresponding to these proteins were excised from the gels, dissolved in $30 \%$ $\mathrm{H}_{2} \mathrm{O}_{2}$, and then counted in a liquid-scintillation counter. The radioactivity in each variant is expressed relative to the total radioactivity for all 5 variants. The results from 2 cultures treated with DMSO and 2 treated with taxol are shown. These data confirm quantitatively that the pattern of chartin variants is altered in taxoltreated neurons compared to control neurons (see text for additional details).

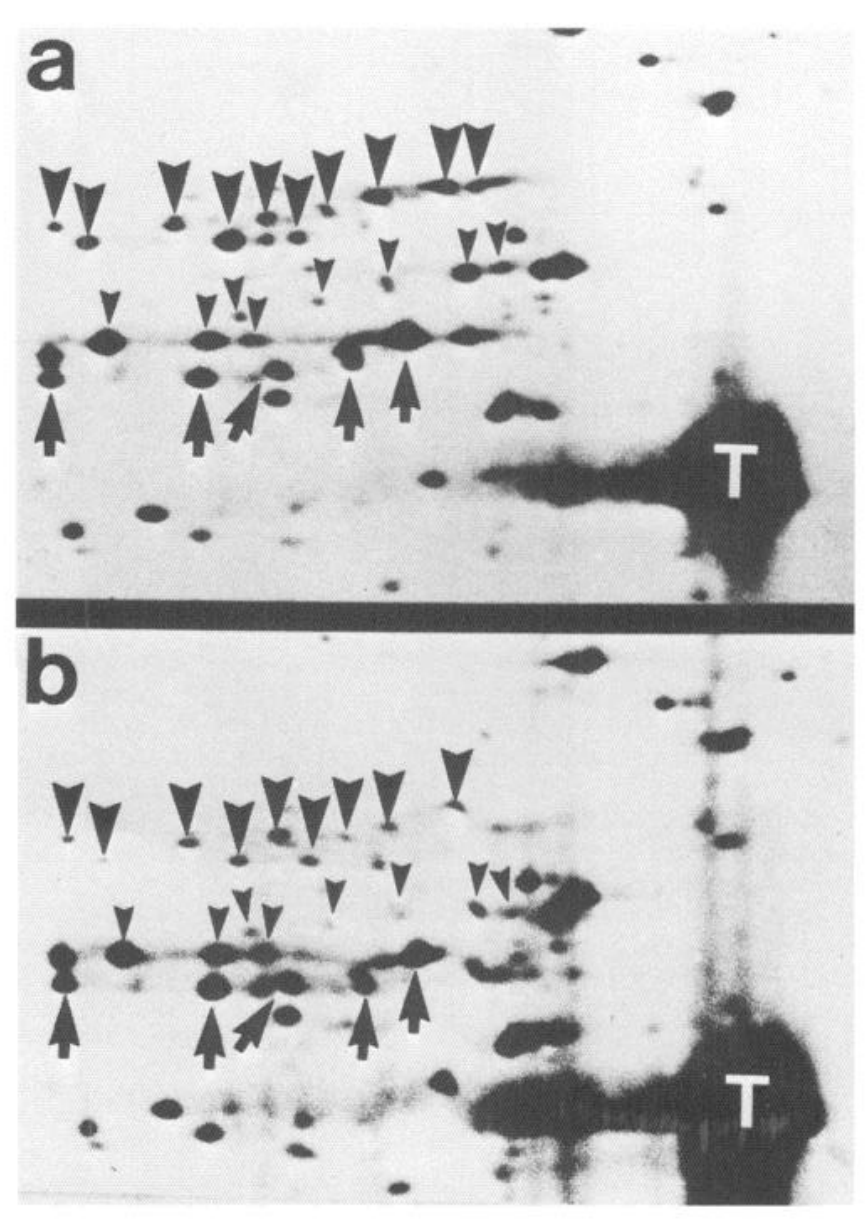

Figure 4. Effects of taxol on chartins synthesized prior to drug treatment. Cultures were labeled for $20 \mathrm{hr}$, incubated in unlabeled medium with either DMSO or taxol for an additional $24 \mathrm{hr}$, and then fractionated for microtubules. Two-dimensional gel profiles of microtubule fractions from DMSO- $(a)$ or taxol- $(b)$ treated cultures are shown. The fluorographs were exposed for $12 \mathrm{~d}$. The data shown are representative of 2 experiments.

scribed results is that taxol interferes with the incorporation of chartins into microtubules. This interpretation is further supported by the observation that taxol interferes with the phosphorylation of the chartins. Chartins are normally multiply phosphorylated in situ (Pallas and Solomon, 1982; Black and Kurdyla, 1983). The generation of the most highly phosphorylated chartin variants is dependent on their incorporation into microtubules (Pallas and Solomon, 1982; Aletta and Greene, 1987). If taxol interferes with the incorporation of chartins into microtubules, then the generation of these more highly phosphorylated, polymer-specific variants should be suppressed in the presence of the drug. This, in fact, was observed qualitatively (Fig. 2) as well as quantitatively for the $64 \mathrm{kDa}$ chartins (Table 1). The more highly acidic variants of each chartin family, which correspond to their highly phosphorylated forms (Pallas and Solomon, 1982; Black and Kurdyla, 1983; Black et al., 1986a), are depleted in taxol-treated, as compared to control cultures, and this decrease is accompanied by a corresponding increase in the relative abundance of the more basic chartin variants.

In the preceding experiments, exposure to taxol coincided with the labeling period. Thus, the observed effects of taxol apply only to chartins synthesized during exposure to the drug. To 


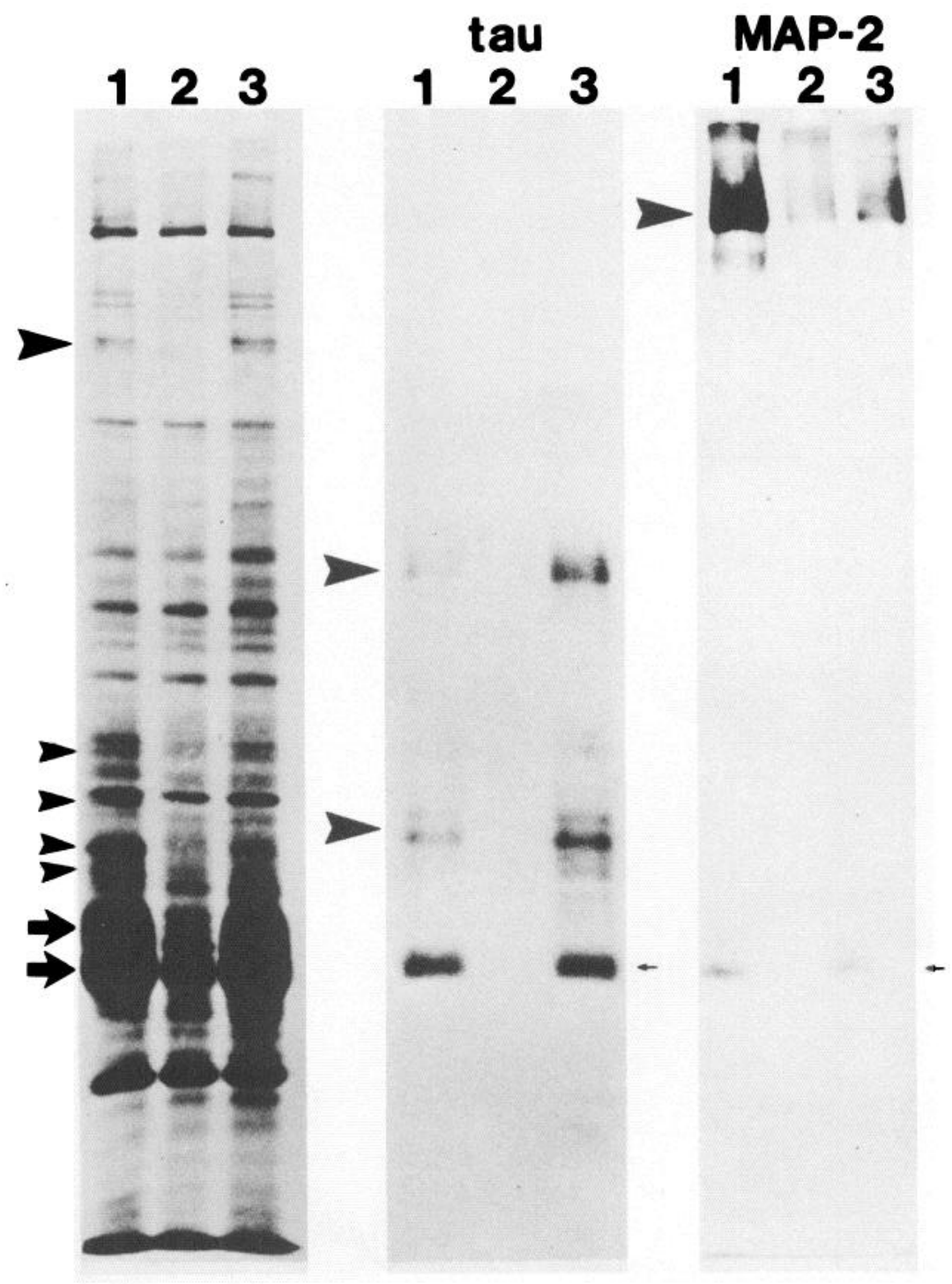

Figure 5. Effects of taxol on other MAPs. Cultures were labeled for $20 \mathrm{hr}$ and treated with either DMSO, podophyllotoxin, or taxol coincident with labeling. Microtubule fractions were prepared from the cultures and analyzed for chartins and the $210 \mathrm{kDa}$ MAP (lefthand panel) directly on ID gels, or for tau and MAP-2 by immunoprecipitation (middle and right-hand panels, respectively). In all panels, lanes $1-3$ depict the material from the DMSOtreated, podophyllotoxin-treated, or taxol-treated cultures, respectively. In the left-hand panel, tubulin is indicated with arrows, the chartins are indicated with small arrowheads, and the $210 \mathrm{kDa}$ MAP with a large arrowhead. The data shown are representative of 6 experiments. The arrowheads by the middle and right-hand panels depict the material that was specifically precipitated by the antibodies, while the small arrow identifies a labeled species that is nonspecifically precipitated and corresponds to $\beta$-tubulin. The immunoprecipitation data are representative of 2 experiments.

determine the possible effects of taxol on chartins that were synthesized and assembled prior to exposure to taxol, neuron cultures were labeled for $18-24 \mathrm{hr}$ and then chased for $24 \mathrm{hr}$ in the presence of DMSO or taxol. Under these conditions, taxol had relatively little effect on the levels of labeled chartins in microtubule fractions (Fig. 4). The difference between the results obtained when labeling is coincident with taxol treatment and when it precedes taxol treatment suggests that taxol interferes with the incorporation of unassembled chartins onto microtubules, but has much less effect on chartins already incorporated into microtubules. To further test this possibility, prelabeled neurons were treated with podophyllotoxin for $24 \mathrm{hr}$ and then allowed to recover for $24 \mathrm{hr}$ in the presence of either taxol or DMSO. Electron microscopy confirmed that microtubules formed in cultures that were allowed to recover in the presence of taxol or DMSO (data not shown). As determined by both 1D and $2 \mathrm{D}$ gel electrophoresis, microtubules formed in the absence of taxol had a normal complement of labeled chartins, while microtubules formed in the presence of taxol were, by comparison, deficient in the chartins (data not shown).

\section{Effect of taxol on incorporation of MAP-2, tau, and the MAP of $\mathrm{M}, 210 \mathrm{kDa}$ onto microtubules}

Microtubule fractions were prepared from cultures incubated simultaneously with ${ }^{35} \mathrm{~S}$-methionine and either DMSO, podophyllotoxin, or taxol, and then assayed for MAPs by 1D gel electrophoresis or immunoprecipitation.

The $210 \mathrm{kDa}$ MAP is well-resolved in 1D gels of microtubule fractions, and consists of 2-3 bands (Fig. 5, left-hand panel, large arrowhead; see also Peng et al., 1985). Tau consists principally of a high-molecular-weight species, $M_{\mathrm{r}} 100,000-110,000$, and lesser amounts of a lower-molecular-weight species, $M_{\mathrm{r}} \sim$ 68,000 (Peng et al., 1985); this lower-molecular-weight material is distinct from the chartins with respect to mobility in 2D gels, reactivity with antibodies against tau, and thermostability (Black and Kurdyla, 1983; Peng et al., 1985). Both tau and the 210 $\mathrm{kDa}$ MAP were present in microtubule fractions from DMSOtreated cultures, and were absent or greatly diminished in microtubule fractions prepared from podophyllotoxin-treated cultures (Fig. 5 and Peng et al., 1985). However, unlike chartins, 


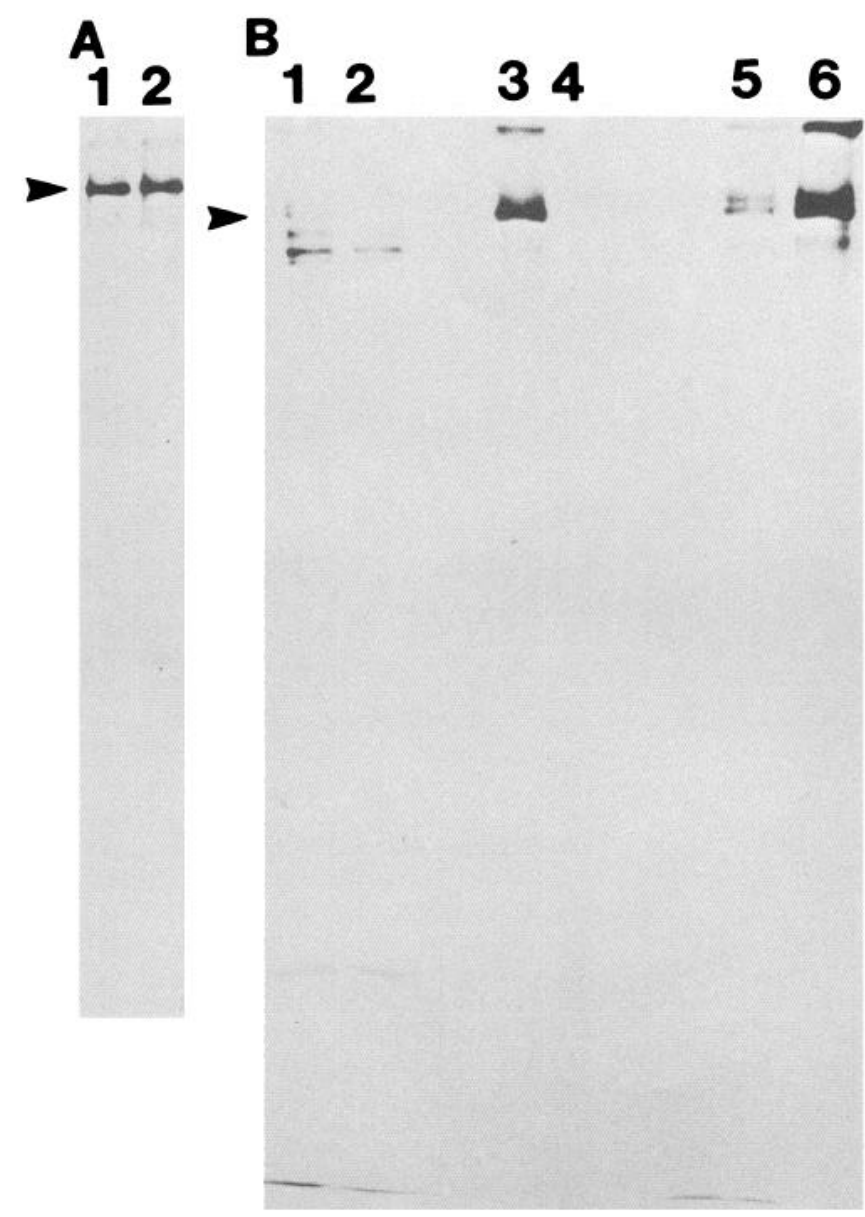

Figure 6. A, Immunoprecipitation of MAP-2 from SDS extracts of sister cultures labeled for $24 \mathrm{hr}$ and treated during the labeling period with either DMSO (lane 1) or taxol (lane 2). Comparable amounts of labeled MAP-2 were precipitated from each culture. $B$, Triton X-100soluble, cold $/ \mathrm{Ca}^{2+}$-soluble and cold $/ \mathrm{Ca}^{2+}$-insoluble fractions were prepared from cultures labeled for $24 \mathrm{hr}$ and treated with either DMSO or taxol during the labeling period, and assayed for labeled MAP-2 by immunoprecipitation. Lanes $I$ and 2 depict immunoprecipitates from Triton X-100-soluble fractions of DMSO- and taxol-treated cultures, respectively. Lanes 3 and 4 depict immunoprecipitates from cold/ $\mathrm{Ca}^{2+}$ soluble fractions of DMSO- and taxol-treated cultures, respectively. Lanes 5 and 6 show immunoprecipitates from cold/ $\mathrm{Ca}^{2+}$-insoluble fractions of DMSO- and taxol-treated cultures, respectively. The arrowhead identifies the position of brain MAP- 2 obtained from immature rats. The specificity of the antibody for precipitating MAP-2 has been established in control experiments using no first antibody or other first antibodies (Peng et al., 1985, and data not shown). The additional bands that migrate just ahead of MAP-2 (these are particularly apparent in the precipitates from the Triton $\mathrm{X}-100$-soluble fraction) are fragments of MAP-2. They are only trace components in precipitates from SDS extracts of cultures (see $A$ ). The data shown are representative of 2 experiments.

tau and the $210 \mathrm{kDa}$ MAP were present in microtubule fractions prepared from taxol-treated cultures, and, notably, at levels closely resembling or possibly a little greater than those in similarly prepared fractions from control cultures (see especially the data for tau in Fig. 5).

The situation for MAP-2 is more complicated. Labeled MAP-2 is prominent in microtubule fractions from control cultures, but it is depleted from corresponding fractions from cultures treated with podophyllotoxin or, surprisingly, taxol (Fig. 5). Labeled MAP-2 accumulates in control and taxol-treated cultures to comparable degrees (Fig. 6A). More detailed analyses of MAP-2 solubility (Fig. 6B) show that most of the labeled MAP-2 of control cultures partitions with the microtubule fraction; much smaller amounts are cold/ $\mathrm{Ca}^{2+}$-insoluble or Triton X-100-soluble. In taxol-treated cultures, almost all of the labeled MAP-2 is cold/ $\mathrm{Ca}^{2+}$-insoluble; very little is Triton X-100-soluble, and even less is apparent in the microtubule fraction.

\section{Discussion}

The present studies have examined the effects of taxol treatment on the interaction of MAPs with microtubules in intact neurons. These analyses relied on a cell extraction assay for obtaining assembled microtubule proteins. The properties of the assay have been established previously (Black et al., 1984, 1986b). It permits quantitative separation of unassembled and assembled microtubule proteins, and also permits separation of the proteins comprising labile microtubules from those of stable microtubules. Labile microtubules are depolymerized by exposure to $\geq 1 \mathrm{~mm} \mathrm{Ca}^{2+}$ and in cultured sympathetic neurons are more abundant than stable microtubules, which are not depolymerized by $\mathrm{Ca}^{2+}$ (Black et al., 1984). Using this assay, we observed that treatment of intact neurons with taxol altered the incorporation of the chartin MAPs and MAP-2 into microtubules, while the levels of tau and the $210 \mathrm{kDa}$ MAP associated with microtubules were unaffected or possibly slightly enhanced.

Chartins are prominent microtubule proteins in untreated and DMSO-treated neurons (Fig. 1). In the sequential extraction assay used here, they partition between unassembled and labile microtubule fractions, and are only trace components in fractions containing stable microtubules (Black and Kurdyla, 1983, and Fig. 3). Neurons exposed to taxol synthesize chartins at apparently normal levels (Fig. 2). However, these chartins do not partition with microtubules (Figs. 1, 3), but remain in the unassembled fraction. These results suggest that taxol interferes with the interaction of chartins with microtubules in intact neurons. This possibility is further supported by the observation that the accumulation of the polymer-dependent phosphorylated variants of the chartins is strongly suppressed in the presence of taxol (Fig. 2, Table 1, and Aletta and Greene, 1987).

The effect of taxol on the interaction of chartins with microtubules is apparently specific for chartins not associated with microtubules at the time of drug exposure. Chartins synthesized and assembled prior to exposure to taxol are present at approximately normal levels on microtubules (Fig. 4). However, when microtubules are first depolymerized and then allowed to repolymerize in the presence or absence of taxol, only microtubules formed in the absence of taxol have chartins. These observations suggest that taxol interferes specifically with the incorporation of chartins into microtubules, but that it apparently does not strongly promote the dissociation of chartins already incorporated into microtubules at the time of drug exposure.

Taxol may interfere with chartin-microtubule interaction by preventing their assembly onto microtubules altogether. It is also possible that chartins may assemble onto microtubules in the presence of taxol, but that their association with the tubulin lattice may be altered such that they very rapidly dissociate from microtubules in situ. It is not possible at present to distinguish between these alternatives. What is clear, however, is that microtubules formed in the presence of taxol are deficient in the chartins. Taxol may interfere with the incorporation of chartins into microtubules by blocking the site(s) on the polymer 
to which the chartins bind. In this regard, taxol has specific binding sites on the tubulin molecule (Parness and Horwitz, 1981). It is also possible that taxol binds directly to chartins and thereby interferes with their assembly onto microtubules.

Taxol also affects the behavior of MAP- 2 in the extraction assay for assembled microtubule proteins, but in a manner that differs from its effects on chartins. In untreated neurons, MAP-2 synthesized during a $24 \mathrm{hr}$ labeling period partitions primarily with labile microtubules (i.e., the cold $/ \mathrm{Ca}^{2+}$-soluble fraction) (Fig. 6). In contrast, MAP-2 synthesized by neurons exposed to taxol partitions almost quantitatively with the cold $/ \mathrm{Ca}^{2+}$ insoluble fraction. This shift in the partitioning of MAP-2 from cold/ $\mathrm{Ca}^{2+}$-soluble to -insoluble fractions occurs with only a minimal shift in the partitioning of tubulin (Fig. 3). Thus, the effect of taxol on MAP-2 is apparently not secondary to a shift in the partitioning of tubulin. The cold $/ \mathrm{Ca}^{2+}$-insoluble fraction is structurally complex; it contains a variety of cytoskeletal components, including stable microtubules, neurofilaments, and microfilaments (Black et al., 1984), as well as other structures. MAP-2 can bind to all of these cytoskeletal structures (Sattilaro et al., 1981; Griffith and Pollard, 1982; LeTerrier et al., 1982; Runge and Williams, 1982) and may also be able to bind other components of the cold $/ \mathrm{Ca}^{2+}$-insoluble fraction. Thus, MAP-2 synthesized in the presence of taxol may preferentially bind to one or more of these components of the cold $/ \mathrm{Ca}^{2+}$-insoluble fraction, rather than to the cold $/ \mathrm{Ca}^{2+}$-labile microtubules with which it normally associates.

The finding that taxol interferes with the incorporation of MAPs into microtubules in intact neurons is unexpected, in view of the observations that the well-characterized MAPs, such as MAP-2, assemble onto microtubules in vitro in the presence of taxol (Kumar, 1981; Vallee, 1982). The fact that taxol selectively interferes with the interaction of some, but not all, MAPs with microtubules argues that its effects are not a manifestation of a generalized toxic reaction to the drug. A simple explanation for the difference between the effects of taxol on MAP-2 interaction with microtubules in vivo and those in vitro is not readily apparent. However, it may be relevant that microtubules assembled in vitro typically have 14 protofilaments (Pierson et al., 1978; McEwen and Edelstein, 1980; Evans et al., 1985), while microtubules assembled in vivo typically have 13 protofilaments (Tilney et al., 1973). Also, in intact cells, but not under the in vitro conditions typically used to prepare microtubule proteins, other structures are present that may effectively compete with microtubules for MAPs in the presence of taxol.

The present studies provide pharmacologic evidence in support of a role for MAPs in microtubule spacing in situ. Taxol strongly promotes the assembly of tubulin in a variety of intact cells (see, for example, Schiff and Horwitz, 1980; De Brabander et al., 1981), including neurons (Masurovsky et al., 1983; Letourneau and Ressler, 1984). In neurons, the resulting microtubules are organized into compact bundles in which the individual microtubules are very tightly packed. In fact, the spacing between neighboring microtubules in these bundles is much closer than that normally observed between adjacent microtubules (Letourneau and Ressler, 1984). Thus, taxol interferes with the regulation of microtubule spacing in situ, in addition to its well-documented microtubule assembly and stabilizing activities. We have shown here that taxol interferes with the normal interaction of at least some MAPs with microtubules in situ. In vitro studies have provided compelling evidence that MAPs contribute to the degree of spacing between neighboring micro- tubules (Herzog and Weber, 1978; Kim et al., 1979; Vallee and Davis, 1983; Brown and Berlin, 1985; Black, 1987). These considerations raise the possibility that the unusually close spacing between microtubules in taxol-treated neurons results from taxol's interference with the interaction of MAPs, such as that of MAP-2 and the chartins with microtubules.

As indicated in the introduction, the spacing between neighboring microtubules is tightly regulated in neurons, so that in axons, microtubules are more closely spaced than in dendrites. Microtubules in these compartments also differ in the MAPs (reviewed in Black and Smith, 1987). Specifically, MAP-2 is enriched on dendritic microtubules, in contrast to those in axons, while the converse is true for tau and the $210 \mathrm{kDa}$ MAP. Also, the phosphorylation state of the chartin MAPs, and possibly MAP- $1 \mathrm{a}$ and MAP- $1 \mathrm{~b}$, in axons differs from that in dendrites. We have suggested that the regional differences in microtubule organization reflect, at least in part, underlying differences in MAPs. The present results fully support this possibility by providing evidence in intact neurons that MAPs are indeed involved in regulating microtubule spacing. Also important in this context is the observation that MAPs differ quantitatively in their effects on the packing density of microtubules, as assayed in vitro (Herzog and Weber, 1978; Black, 1987). Thus, the segregation of MAPs into either the axonal or the dendritic compartment represents a mechanism for generating regional differences in the internal structure of the neuron. This, in turn, may contribute to the well-documented regional differences in neuronal form and function.

\section{References}

Aletta, J. M., and L. A. Greene (1987) Sequential phosphorylation of chartin proteins is regulated by the presence of microtubules. J. Cell Biol. 105: 277-290.

Bartlett, W. P., and G. A. Banker (1984) An electron microscopic study of the development of axons and dendrites by hippocampal neurons in culture. II. Synaptic relationships. J. Neurosci. 4: 1954 1965.

Binder, L. I., A. Frankfurter, and L. I. Rebhun (1985) The distribution of tau in the mammalian central nervous system. J. Cell Biol. 101: 1371-1378.

Black, M. M. (1987) Comparison of the effects of MAP-2 and tau on the packing density of microtubules. Proc. Natl. Acad. Sci. USA (in press).

Black, M. M., and J. T. Kurdyla (1983) Microtubule-associated proteins of neurons. J. Cell Biol. 97: 1020-1028.

Black, M. M., and I. Peng (1985) In vivo taxol treatment alters the solubility properties of microtubule-associated proteins. Ann. NY Acad. Sci. 466: 426-428.

Black, M. M., and W. Smith (1987) Regional differentiation of the neuronal cytoskeleton. Appendix: Diffusion of proteins in the neuron cell body: Mathematical approximations and computer simulations. In Intrinsic Determinants of Neuronal Form and Function, R. J. Lasek and M. M. Black, eds. Alan R. Liss, NY.

Black, M. M., J. M. Cochran, and J. T. Kurdyla (1984) Solubility properties of tubulin in developing neurons: Evidence for labile and stable microtubules. Brain Res. 295: 255-263.

Black, M. M., J. M. Aletta, and L. A. Greene (1986a) Regulation of microtubule composition and stability during nerve growth factorpromoted neurite outgrowth. J. Cell Biol. 103: 545-557.

Black, M. M., P. Keyser, and E. Sobel (1986b) Interval between the synthesis and assembly of cytoskeletal proteins in cultured neurons. J. Neurosci. 6: 1004-1012.

Bonner, W. M., and R. A. Laskey (1974) A film detection method for tritium-labeled proteins and nucleic acids in polyacrylamide gels. Eur. J. Biochem. 46: 83-88.

Brown, P. A. and R. D. Berlin (1985) Packing volume of sedimented microtubules: Regulation and potential relationship to an intracellular matrix. J. Cell Biol. 101: 1492-1500. 
Caceres, A., L. I. Binder, M. R. Payne, P. Bender, L. Rebhun, and O. Steward (1984) Differential subcellular localization of tubulin and the microtubule-associated protein MAP-2 in brain tissue as revealed by immunocytochemistry with monoclonal hybridoma antibodies. J. Neurosci. 4: 394-410.

Collins, C. A., and R. B. Vallee (1986) Reversible assembly cycling of taxol stabilized microtubules. J. Cell Biol. 103: 403a

De Brabander, M., G. Geuens, R. Nuydens, R. Willebrords, and J. De Mey (1981) Taxol induces the assembly of free microtubules in living cells and blocks the organizing capacity of the centrosomes and kinetochores. Proc. Natl. Acad. Sci. USA 78: 5608-5612.

DeCamilli, P., P. Miller, F. Navone, W. E. Theurkauf, and R. B. Vallee (1984) Distribution of microtubule-associated protein 2 (MAP2) in the nervous system of the rat studied by immunofluorescence. Neuroscience 11:819-846.

Evans, L., T. Mitchinson, and M. Kirschner (1985) Influence of the centrosome on the structure of nucleated microtubules. J. Cell Biol. 100: 1185-1191.

Griffith, H., and T. D. Pollard (1982) The interaction of actin filaments with microtubules and microtubule-associated proteins. J. Biol. Chem. 27: 9143-9151.

Herzog, W., and K. Weber (1978) Fractionation of brain microtubuleassociated proteins. Isolation of two different proteins which promote tubulin polymerization in vitro. Eur. J. Biochem. 92: 1-8.

Huber, G., and A. Matus (1984) Differences in the cellular distribution of two microtubule-associated proteins, MAP-1 and MAP-2, in rat brain. J. Neurosci. 4: 151-161.

Kim, H., L. I. Binder, and J. L. Rosenbaum (1979) The periodic association of $\mathrm{MAP}_{2}$ with brain microtubules in vitro. J. Cell Biol. 80: $266-276$.

Kumar, N. (1981) Taxol-induced polymerization of purified tubulin. J. Biol. Chem. 256: 10435-10441.

Langford, G. M. (1983) Length and appearance of projections on neuronal microtubules in vitro after negative staining: Evidence against a crosslinking function for MAPs. J. Ultrastruct. Res. 85: 1-10.

LeTerrier, J. F., R. K. H. Liem, and M. Shelanski (1982) Interactions between neurofilaments and microtubule-associated proteins: A possible mechanism for intra-organelle bridging. J. Cell Biol. 95: 982986.

Letourneau, P. C., and A. H. Ressler (1984) Inhibition of neurite initiation and growth by taxol. J. Cell Biol. 98: 1355-1362.

Magendantz, M., and F. Solomon (1985) Analyzing components of microtubules: Antibodies against chartins, associated proteins from cultured cells. Proc. Natl. Acad. Sci. USA 82: 6581-6585.

Masurovsky, E. B., E. R. Peterson, S. M. Crain, and S. B. Horwitz (1983) Morphological alterations in dorsal root ganglion neurons and supporting cells of organotypic mouse spinal cord-ganglion cultures exposed to taxol. Neuroscience 10:491-509.

McEwen, B., and S. J. Edelstein (1980) Evidence for a mixed lattice in microtubules reassembled in vitro. J. Med. Biol. 139: 123-145.

Pallas, D., and F. Solomon (1982) Cytoplasmic microtubule-associated proteins: Phosphorylation at novel sites is correlated with their incorporation into assembled microtubules. Cell 30:407-414.

Parness, J., and S. B. Horwitz (1981) Taxol binds to polymerized tubulin in vitro. J. Cell Biol. 91: 479-487.

Peng, I., L. I. Binder, and M. M. Black (1985) Cultured neurons contain a variety of microtubule-associated proteins. Brain Res. 361: 200211

Peng, I., L. I. Binder, and M. M. Black (1986) Biochemical and immunological analyses of cytoskeletal domains of neurons. J. Cell Biol. 102: 252-262.

Pierson, G. B., R. E. Hinkley, and R. H. Himes (1978) Alterations in number of protofilaments in microtubules assembled in vitro. J. Cell Biol. 76: 223-228.

Runge, M., and R. C. Williams (1982) Formation of an ATP-dependent microtubule-neurofilament complex in vitro. Cold Spring Harbor Symp. Quant. Biol. 46: 483-493.

Sattilaro, R. F., W. L. Dentler, and E. L. LeCluyse (1981) Microtubuleassociated proteins (MAPs) and the organization of actin filaments in vitro. J. Cell Biol. 90: 467-473.

Schiff, P. B., and S. B. Horwitz (1980) Taxol stabilizes microtubules in mouse fibroblast cells. Proc. Natl. Acad. Sci. USA 77: 1561-1565.

Solomon, F., M. Magendantz, and A. Salzman (1979) Identification with cellular microtubules of one of the co-assembling microtubuleassociated proteins. Cell 18: 431-438.

Tilney, L. G., J. Bryan, D. J. Bush, K. Fujiwara, M. W. Mooseker, D. B. Murphy, and D. H. Snyder (1973) Microtubules: Evidence for 13 protofilaments. J. Cell Biol. 59:267-275.

Vallee, R. B. (1982) A taxol-dependent procedure for the isolation of microtubules and microtubule-associated proteins (MAPs). J. Cell Biol. 92: 435-442.

Vallee, R. B., and S. E. Davis (1983) Low molecular weight microtubule-associated proteins are light chains of microtubule-associated protein 1 (MAP1). Proc. Natl. Acad. Sci. USA 80: 1342-1346.

Wuerker, R. B., and J. B. Kirkpatrick (1972) Neuronal microtubules, neurofilaments, and microfilaments. Int. Rev. Cytol. 33: 45-75.

Zingsheim, H.-P., W. Herzog, and K. Weber (1979) Differences in surface morphology of microtubules reconstituted from pure brain tubulin using two different microtubule-associated proteins: The high molecular weight MAP 2 proteins and tau proteins. Eur. J. Cell Biol. 19: $175-183$. 